\title{
De l'Amazonie Brésilienne aux Musées Français: parcours de collections et processus de légitimation
}

\author{
Anouk Delaître \\ Pascale de Robert ${ }^{\mathrm{b}}$
}

Para preparar uma pesquisa colaborativa sobre coleções museais amazónicas conservadas na França, foi realizado um inventário no Musée do Quai Branly e no Muséum de Toulouse. Foram identificadas 15 coleções com objetos associados ao povo Mebêngôkre-Kayapó que podiam interessar os pesquisadores indígenas o apresentar itens com documentação incompleta ${ }^{1}$. A análise da documentação das coleções, juntamente com a história das coleções, é uma oportunidade para um estudo comparativo e cronológico das políticas de aquisições referentes aos dois museus. Desde o século XIX, destacam-se pessoas-chaves, colecionadores e outros, suscetiveis de influenciar os processos de constituição, legitimação e documentação das coleções museais. Com que objetivos se manifestam em cada época? O caso amazônico ilustra os processos de legitimação operados pela instituição do museu, e a importância de contextualizar as condições de aquisição do ponto de vista institucional, político e jurídico. As iniciativas mais recentes tentam valorizar narrativas múltiplas para associar aos objetos das coleções.

Estudos em museus, Coleções etnográficas, Documentação participativa, Estudo de proveniência, Política de aquisição.

Centré sur des ateliers de documentation participative, le projet COLAM a réuni une délégation franco-brésilienne (étudiants, scienti-

a Doctorante en Anthropologie Sociale, ED TESC, Université Toulouse - Jean Jaurès. Email: anouk.delaitre@univ-tlse2.fr.

b Anthropologue, UMR PALOC-IRD/MNHN. Email: pascale.derobert@ird.fr. 
fiques, experts amérindiens, professionnels du patrimoine) autour de collections des basses terres amazoniennes conservées en France (Fernando Baniwa 2018). Pour présenter des objets pertinents à expertiser aux yeux de chacun, un travail de sélection et de recherche mené en amont des ateliers s'avère essentiel ${ }^{2}$; il passe nécessairement par une étude approfondie de la documentation et de l'histoire des collections inventoriées pour l'occasion (Delaître 2017).

Cet article présente une partie des réflexions et du processus muséologique mis en place avant les ateliers COLAM dans le cadre d'une recherche sur la constitution et les provenances des collections qui sont des questions prégnantes dans l'actualité muséale européenne ${ }^{3}$. Notre intérêt se porte sur les modes de circulation à l'origine des collections patrimoniales, à savoir les parcours empruntés par les objets, mais aussi les personnes qui les sélectionnent et les transportent ainsi que les connaissances dont ils sont les médiateurs. La réflexion est menée à partir des collections des Mebêngôkre-Kayapó inventoriées dans deux musées français. En comparant les personnalités liées à leurs constitutions à différentes époques, on peut suivre une métamorphose de la figure du collecteur (missionnaire, naturaliste, ethnographe, galeriste, muséologue...) chaque fois ancré dans un réseau déterminé, et jouant un rôle déterminant sur la collection et les récits qui y sont reliés. Chacun présente un regard et un discours propre, faisant respectivement figure d'expert, tour à tour reconnu par l'institution. Leur nom demeure au sein de la documentation et suit ainsi l'objet dans sa vie au musée. L'étude de ce phénomène de légitimation permet de saisir, au-delà du fossé entre musées ethnographiques et d'art, la place passée, actuelle et en devenir, accordée aux savoirs 'autres' dans le musée, non seulement ceux descautochtones mais plus largement aux savoirs non-institutionnels.

\section{Ouvrir les musées, raconter les collections : perspectives théoriques}

Le musée, en tant que gardien d'un patrimoine public, est par sa fonction un lieu de conservation et de transmission. En écho à la 
« zone de contact "(Clifford 1997), ses gestionnaires sont appelés à œuvrer pour ce faire en partenariat avec la société civile. Dans le cas des musées disposant de collections extra-occidentales, liées à la représentation des sociétés d'ailleurs, les héritiers concernés par ce patrimoine prennent place sur une plus grande échelle géographique. Faisant face à la question de la reconnaissance, les pratiques muséales à leur égard démontrent des défis politiques, éthiques, épistémologiques et méthodologiques. La volonté de décoloniser les collections implique de continuer à repenser les politiques muséales dans une perspective de prise en compte et d'échanges avec les populations-sources. Outre les restitutions matérielles, il peut s'agir de prêts d'objets, projets communs, consultation, expertises muséales ; l'ensemble étant placé dans une logique d'ouverture développée à partir des collections. Ces expériences se multiplient désormais avec des modalités différentes et de façon plus ou moins systématique selon les pays, comme au Brésil où de nombreux projets ont déjà été menés ${ }^{4}$.

Les collections ethnographiques sont encore une opportunité pour réfléchir à nos rapports aux objets et aux relations que chaque société noue avec les autres. Bien que le Musée de l'Autre soit distingué du Musée du Soi (L'Estoile 2007), les collections qui le composent dénotent d'un passé commun et de relations tissées. L'étude biographique des objets, en retraçant leurs trajectoires, identifie leurs potentiels changements de statuts et les révèle comme support de vie sociale (Appadurai 1986). L'objet muséalisé devient sémiophore (Pomian 1996), c'est-à-dire porteur de sens. Il témoigne d'une polysémie dès lors qu'il est appréhendé dans son historicité complète (Bonnot 2014), avant et au sein du musée. En tant que médiateur de connaissances, il est de ce fait assujetti à un phénomène de mises en récit mouvant au long de sa vie muséale. "Aucun objet n'a de statut définitif. Personne ne peut décréter s'il relève du document ethnographique ou de l'art contemporain. La vie de l'objet échappe à ses fabricants comme à ses acheteurs, aux guerriers papous comme aux galeristes, à l'ethnologue comme au commissaire d'exposition. » 
(Bensa 2006:156). Dans le cas des collections ethnographiques, l'enjeu est d'identifier, étudier et croiser les différents regards qu'elles ont suscité ou suscitent encore de manière à encourager la mise en place de dialogues au sein de l'espace muséal.

\section{Une étude de cas pratique: les collections des basses terres amazoniennes en France}

A l'initiative d'une rencontre muséale autour d'objets de collections botaniques et ethnographiques, le projet COLAM visait d'abord à présenter un matériel deétude exploitable, soit des objets à la documentation lacunaires pertinents à expertiser pour les représentants amérindiens invités, mais aussi les responsables des collections. Il nécessitait donc en amont d'effectuer l'inventaire et l'étude de la documentation de collections des basses terres amazoniennes. Au printemps 2018, la délégation franco-brésilienne a été accueillie au Musée du quai Branly - Jacques Chirac par Fabienne de Pierrebourg, chargée des collections Amérique, et au Muséum de Toulouse par Sylviane Bonvin-Pochstein, chargée des collections d'ethnographie. C'est au sein de ces deux institutions - aux héritages et conjonctures propres - que les collections amazoniennes, sélectionnées en donnant une priorité à celles constituées d'objets du Moyen Xingu et du Rio Negro ${ }^{5}$, ont été recensées.

Pour comprendre la constitution de la documentation et l'intérêt muséal qu'elle reflète en tant que valeur patrimoniale, il est impératif d'opérer un travail de déconstruction en revenant sur l'histoire des collections. L'enjeu est d'identifier et de contextualiser tant les statuts attribués aux collections que les regards qui en sont à l'origine pour faire apparaître au final les différents récits - de la constitution à la muséalisation - qu'elles ont suscité et suscitent encore.

Dans ce cadre, notre étude s'est d'abord focalisée sur les politiques, passées et actuelles, d'enrichissement concernant les objets venus d'ailleurs et muséalisés. Ceci d'autant plus qu'au musée, l'enrichissement des collections peut être compris dans sa dimension ma- 
térielle, via l'acquisition de nouveaux fonds, ou immatérielle, via la révision de la documentation des collections.

En identifiant et contextualisant les voix apposées aux objets amérindiens conservés, l'enjeu est de démontrer l'importance jouée par l'individu lié à l'arrivée des objets au musée, qu'il soit collectionneur ou non. On s'attache à reconnaitre les parcours empruntés et les réseaux développés pour que l'objet vienne au musée, et montrer leurs influences dans la perception actuelle de la qualité patrimoniale des collections conservées. L'intérêt est porté sur les modes de circulation dans lesquels objets, personnes et savoirs sont inscrits. Les figures d'experts identifiés laissent voir comment s'est transformée, au cours de l'histoire, la place laissée aux savoirs non-académiques dans les musées.

Empruntée à Virginie Soulier (2013), l'analyse de la construction des discours est transposée de l'exposition à la documentation des collections, qui est un discours normalisé selon des logiques propres à chaque institution, parfois plurielles selon les politiques appliquées par les gestionnaires qui se sont succédés au sein même de l'institution. La documentation accompagne l'objet tout au long de sa vie au musée : en le renseignant, elle justifie sa présence et participe à la définition de sa valeur patrimoniale. Pour retrouver l'acte individuel et son empreinte dans ce processus de patrimonialisation, il s'agit de comprendre " comment l'attachement des individus aux choses constitue la clé de leur devenir" (Bonnot 2014:178). La biographie d'objet est appliquée à la lumière des personnes qui participent à la mise en collection afin « de ne pas évacuer de l'enquête les subjectivités, les affects de ceux qui manipulent, s'approprient, désirent les objets, y compris des chercheurs eux-mêmes " (Bonnot 2014:179).

Le travail s'est effectué en commençant par l'inventaire des collections concernées par le projet, puis l'étude de la documentation liée et la recherche sur l'histoire des collections afin de pouvoir effectuer la sélection desdits objets à expertiser. En raison de la participation de 
chercheurs amérindiens Mêbêngokre-Kayapó au sein du projet COLAM, c'est d'abord sur les collections susceptibles de les intéresser directement que nous nous sommes penchées. Les deux institutions visitées ayant des héritages, conjonctures et modes de fonctionnement différents, nous avons été amenées à ajuster la méthodologie au vu de leurs spécificités. L'histoire des collections a été renseignée à travers les informations obtenues aux services d'archives des deux musées concernés et, quand cela était possible, par le biais d'entretiens auprès des personnes chargées des collections, ou associées de près ou de loin à leur constitution. L'ensemble a fourni un matériel d'étude hétéroclite (fiches documentaires, archives, entretiens enregistrés et transcris, notes de lectures, accompagnement d'une visite de réserve, site internet de projets et institutions publiques, etc.) à la base d'un mémoire de recherche en muséologie (Delaître 2017).

\section{Inventaire et typologie des collections: une étape clé en vue d'ateliers collaboratifs}

En 1999, 2800 pièces provenant des basses terres amazoniennes ont été dénombrées ${ }^{6}$ dans les collections françaises de 53 musées et institutions du territoire national (Mongne 1999). Le Musée de l'Homme n'en faisant pas partie, aucun inventaire similaire n'avait été fait à notre connaissance au sujet des collections américanistes conservées au Musée du quai Branly - Jacques Chirac.

Or ce dernier compte douze collections constituées d'objets liés aux catégories " kayapo " et assimilées. Celles-ci sont classées par ordre d'arrivée et modes d'acquisition (don, mission, achat) : la plus ancienne date de 1930, époque du Musée d'Ethnographie du Trocadéro. Huit autres sont arrivées ensuite au Musée de l'Homme : une ré-identifiée en 1949, un don du Museu Paraense E. Goeldi (Belém, Brésil) en 1950, une vente doublée d'un don particulier en 1964 et 1965, une mission en 1971, un don particulier en 1980, un achat en galerie ainsi qu'à un particulier en 1989. Dans l'entredeux muséal, un achat est effectué auprès d'une galerie en 2003. 
Suite à l'ouverture du Musée du Quai Branly - Jacques Chirac qui va hériter des principales collections conservées jusque-là au Musée de l'Homme, une préemption aux enchères est faite en 2007, un achat auprès d'un particulier en 2008, et un don d'un leader amérindien est reçu en 2010 .

Ce corpus conséquent a permis de dérouler un fil historique illustrant les métamorphoses vécues par les collections. Il s'est avéré intéressant de le mettre en regard avec les objets inventoriés dans la deuxième institution visitée : le Muséum d'Histoire Naturelle de Toulouse. Ce dernier est à la tête de trois collections qui étaient susceptibles d'intéresser les Mebêngôkre-Kayapó dont une plus récente issue d'une campagne de collecte contemporaine achevée en 2016, et une autre très ancienne acquise à travers le don d'un particulier et datée de 1911.

On reconnaît dans le contenu de ces collections, l'attrait occidental pour l'exotisme qui a suscité l'importation d'objets représentatifs des populations amérindiennes selon l'imaginaire collectif d'alors. La fascination pour 'l'Indien', véritable guerrier ou bon sauvage selon les périodes, prend matériellement forme par l'importation de plumasserie et d'armes (Mongne 2013). Ces objets constituent les premières collections ramenées aux musées. Il faut d'ailleurs attendre le $\mathrm{XX}^{\mathrm{e}}$ siècle pour que la typologie des collections se diversifie, via l'affirmation d'une ethnologie visant à connaître une société de manière exhaustive à travers sa culture matérielle (Pierrebourg \& Sevilla 2010).

Au sein de ce découpage chronologique, l'analyse du contenu des collections, de leurs modes d'acquisition - reflétant des besoins et contingences propres aux institutions-hôtes - et de leur documentation fait apparaître, trois types de collection qualifiés de façon préliminaire selon un prisme contextuel : scientifique, esthétique et participatif (Figure 1). 


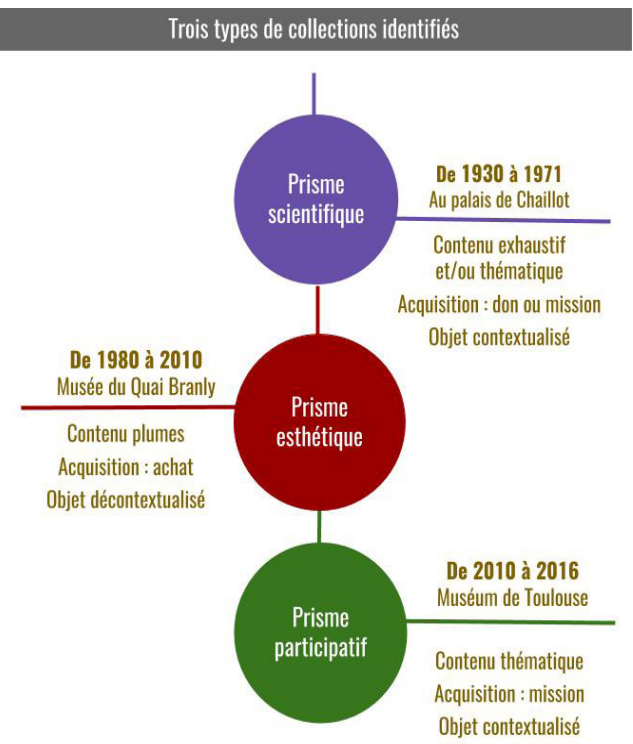

Figure 1 : Typologie schématique des collections étudiées (Source : Delaître, $2017: 80$ )

Au Musée du quai Branly, deux types de collections se distinguent : celles issues de don ou mission opérés jusqu'en 1971 et celles issues d'achats réalisés depuis 1980. Le premier regroupe les collections arrivées entre 1930 et 1971 au Palais de Chaillot, par don ou mission de collecte. Héritier des collections du Musée d'Ethnographie du Trocadéro, le Musée de l'Homme est inauguré en 1938 sous le modèle du musée-laboratoire impulsé par Paul Rivet et Georges-Henri Rivière (Blanckaert 2015). C'est le temps du 'paradigme muséal' (L'Estoile 2007), le musée et l'ethnologie ne font qu'un ; le premier servant à asseoir la légitimité de la seconde. Le Musée de l'Homme est alors inséré dans une tradition anthropologique 'scientifiquement périmée' combinant l'étude des caractères physiques avec celles des faits culturels (Grognet 2013). Les sociétés extra-occidentales sont étu- 
diées à partir de leurs productions matérielles. A la base de toute démarche ethnologique, l'acquisition de collections est idéalement issue de missions scientifiques, sinon de dons ou prêts inter-muséaux (par exemple le don du Museu Goeldi). Fidèles au principe de collecte tel que décrit dans le Manuel d'ethnographie de Marcel Mauss (1926), les contenus exhaustifs des collections kayapo étudiées témoignent de cette logique.

Les objets sont 'contextualisés' par la documentation au travers d'un système de fiche à 10 points listant lieu d'origine ; dénomination et nom ; description et matière ; fabrication, usage et typologie ; ethnographie, peuple ; par qui et quand l'objet a été recueilli ; conditions d'entrée au musée, don, achat, prêt, dépôt ; référence photographique et iconographiques; références muséographiques; bibliographie, date et rédaction de la fiche. L'ensemble est conservé dans la documentation actuelle retrouvée dans la base de données 'The Museum System Objects - TMS objets' du Musée du quai Branly - Jacques Chirac (Beltrame 2012).

Le second type de collections distingué est représenté par des pièces arrivées entre 1980 et 2010 au Musée de l'Homme et au Musée du quai Branly, majoritairement par achat sur le marché de l'art, ou ponctuellement par don. L'achat traduit une acquisition directe effectuée en regard du contenu existant des fonds patrimoniaux qui doivent être renouvelés. Le contenu des collections, quasi-exclusivement composées de plumasserie, perd de ce fait en exhaustivité et gagne en logique sérielle. Dans la documentation, on constate que l'objet est décontextualisé : seuls les renseignements principaux demeurent (désignations, dimensions, matériaux...) qui traduisent les intérêts muséaux de la conservation et de l'exposition. L'ensemble augure le nouveau statut attribué à l'objet devenu œuvre d'art.

Cette distinction entre deux grands types de collections au musée Branly permet aussi une mise en regard avec le Muséum de Tou- 
louse, où la récente campagne de collecte, à visée scientifique et de type participative, retient l'attention. En termes de lignes d'acquisition de fonds extra-occidentaux, la collecte n'est plus une pratique en vigueur, car en partie jugée désuète, par les institutions muséales (qui n'ont souvent plus de lien direct avec les centres de recherches ethnologiques établis dans les universités). Cependant, le marché de l'art n'offre des objets extra-occidentaux que peu renseignés, voire 'muets', et à des prix exacerbés. Sylviane Bonvin-Pochstein, chargée des collections d'ethnographie du Muséum, a décidé de renouer avec le terrain en montant la 'Mission Brésil' pour regagner en pertinence scientifique (Bonvin-Pochstein 2018). Certains aspects de cette démarche toulousaine se rapprochent des missions de collectes mandatées par le Musée de l'Homme à l'époque de Paul Rivet, à la différence d'un contenu non exhaustif et d'un mode de constitution suivant un modèle dit participatif.

\section{Comprendre les politiques d'acquisition selon leurs injonctions contemporaines}

$\mathrm{Au}$ total, chaque période d'acquisition révèle une 'manière de faire' autrement expliquée à la lumière de facteurs externes institutionnel, juridique, politique - impactant la circulation des objets, des personnes et des savoirs associés à la constitution des collections ethnographiques. Les résultats de cette analyse sont représentés sur les frises chronologiques représentées ci-après (Figure 2). A partir de ce schéma, on développe les principaux facteurs susceptibles de faire changer, au cours de l'histoire de ces collections, les statuts et les rôles accordés aux objets amérindiens conservés dans les musées français. 


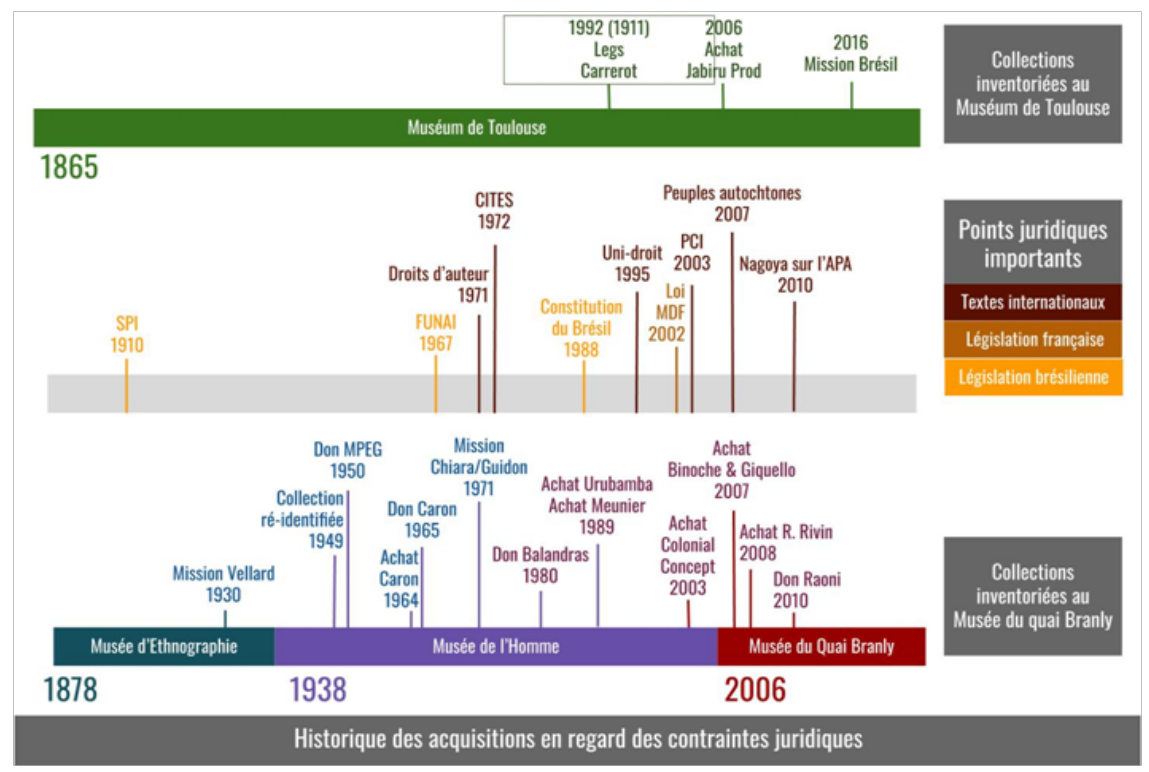

Figure 2: Histoire des collections et évolution des législations pour les musées.

Sur la Figure 2, on a distingué (en bas) une frise chronologique concernant les collections actuellement conservées au Musée du quai Branly - Jacques Chirac (MQB) et ordonnées en fonction de leur date d'enregistrement dans l'institution muséale. Sur la frise chronologique du haut, on a placé les trois collections étudiées au Muséum d'Histoire Naturelle de Toulouse (MT), également ordonnées de la plus ancienne à la plus récente. Des règlementations impactant les collections amazoniennes étudiées sont signalées sur la frise centrale.

Il faut reconnaître que les législations et normes internationales, nationales et locales jouent un rôle important sur le statut des objets gardés en collection, au moins dans les musées. Les principales d'entre elles, pour les collections qui nous intéressent, ont été représentées sur la ligne horizontale centrale de la figure 2. Au Brésil, l'accès aux terres indigènes (soit les territoires amérindiens délimités et reconnus 
comme tels par l'Etat) et l'importation d'objets dits d'artisanat sont réglementés en regard des politiques appliquées depuis le Service de Protection des Indigènes (SPI) remplacé par la Fondation Nationale de l'Indien (FUNAI) en 1967. De 1933 à 1968, un 'Conseil de surveillance des expéditions artistiques et scientifiques' chargé de préserver le patrimoine de la nation brésilienne, devait veiller à ce que toute expédition scientifique menée sur le territoire national soit en mesure de constituer une collection en doublon : si l'une était destinée à l'institution étrangère, alors sa jumelle devait rester au Brésil (Grupioni 1998). Plus tard, la Constitution du Brésil de 1988 met fin aux politiques d'assimilation en reconnaissant en même temps un statut et des droits spécifiques, notamment territoriaux, aux amérindiens (Buchillet 1993). De ce fait les missions scientifiques doivent obtenir une autorisation de la FUNAI pour pénétrer les Terres Indigènes.

En France, la loi du 4 Janvier 2002 harmonise le statut des musées de France ${ }^{8}$ agréés par l'Etat et les rassemble sous un corpus de règles et missions parmi lesquelles "Conserver, restaurer, étudier et enrichir leurs collections " (Art. L441-2). Les politiques de conservation et d'acquisition s'en trouvent normalisées. Les musées gestionnaires de collections appartenant à l'Etat doivent obtenir l'aval d'instances scientifiques pour toute proposition d'acquisition (Art. L451-1). Dans le cas d'une vente publique, l'Etat peut exercer un droit de préemption concernant une proposition d'acquisition préalablement acceptée (Art. L.123-2).

Les conventions internationales veillent à la protection des biens culturels matériels comme immatériels (PCI 2003). Participant de leur reconnaissance patrimoniale, elles imposent désormais que toute acquisition soit prouvée par un certificat d'exportation (Unidroit 1995). Les droits d'auteur (1971) prennent également un nouvel écho avec la reconnaissance des peuples autochtones (2007) dont font partie les populations amérindiennes du Brésil. Le Protocole de Nagoya (2010) visant à modifier les pratiques de recherche et notamment de collecte en regard des droits des populations et pays parte- 
naires, invite à régulariser les collectes d'échantillons et le recueil de connaissances traditionnelles sur la biodiversité associées (Aubertin et Nivart 2017).

Toutes ces mesures, cumulatives, jouent un rôle déterminant sur le type et la composition des collections admises au musée à chaque époque considérée. Ainsi, depuis 1972, l'application de CITES interdisant la vente de biens issus d'espèces animales protégées, impacte indirectement les collections amazoniennes puisqu'elle complexifie drastiquement l'acquisition d'objets à plumes (la plupart des plumes utilisées dans la confection des coiffes d'Amazonie brésilienne proviennent d'espèces de la liste CITES). Du fait du mythe de "l'Indien à plumes » implanté dans l'imaginaire occidental (Mongne 2013), la plumasserie garde une part belle dans les collections américanistes et ceci s'observe déjà parmi les fonds les plus anciens. Les plumes, parmi les matériaux les plus sensibles en termes de conservation, sont soumises à des normes rigoureuses, les empêchant notamment d'être exposées sur une longue durée et nécessitant un système de rotation assez rapide dans l'espace d'exposition. En conséquence, on comprend qu'il y a une forte demande de la part de la communauté muséale désireuse de renouveler des fonds 'vieillissant mal', en même temps qu'une raréfaction des biens sur le marché au vu de normes toujours plus restrictives.

\section{Des objets-personnes, comprendre les influences jouées par les "faiseurs de collections "}

Les collections de nos musées sont souvent affiliées au(x) nom(s) des personnes qui les ont constituées et en ont permis l'acquisition. Parfois inscrits dans la documentation des collections, ces noms de personnes sont ainsi muséalisés avec l'objet rapporté et donnent bien souvent une 'signature' aux artefacts concernés. Renseigner les biographies de ces individus expressément nommés - faisant donc autorité et se superposant parfois complètement à la collection elle-même aide à déterminer la manière dont ils œuvrent sur les collections et 
l'influence qui leur survit. Ces noms sont, presque toujours, ceux de personnes étrangères à la société qui a fabriqué l'objet.

Les institutions muséales sollicitent ou acceptent de collaborer avec des individus aux qualités spécifiques en raison de leur proximité avec les objets et connaissances à leur sujet. Chacun participe à la mise en collection selon une démarche propre et dénote d'une typologie d'objets ramenés selon les intérêts portés à l'objet. Faisant figure d'expert, son autorité est répliquée. Ancré dans une temporalité, le neurelectrônico porte un regard et un discours sur l'objet, lui apposant un statut particulier et contribuant à sa mise en valeur (Boltanski \& Esquerre 2017). Justifiant l'acquisition, le récit construit autour de l'objet est également légitimé par son entrée au musée. L'on assiste à une métamorphose de la figure de l'expert - incluant la plupart du temps des personnes jusque-là extérieures à l'institution - qui influent sur la constitution de collections et participent tour à tour à l'élaboration de leur valeur. Au cours de ce processus, qui n'inclue que partiellement et très récemment, pour les collections étudiées, des experts amérindiens, on observe souvent la réduction à un récit unique qui restera attaché à l'objet muséifié. Alors que l'histoire des collections héritées du passé colonial tend à réduire les récits sur les objets, l'ouverture des musées aux amérindiens pourrait appuyer un processus contraire : réhabiliter et valoriser les voix multiples autour des collections, en incluant celles élaborées par les amérindiens sur leurs objets (Oliveira \& Santos 2019).

\section{Au palais de Chaillot, donner aux collections leurs valeurs scientifiques et éducatives}

Au Musée du quai Branly - Jacques Chirac, la plus ancienne collection inventoriée compte 82 objets identifiés 'Caiapo' associés au nom de Jean Vellard. Elle est enregistrée en 1930 au Musée d'Ethnographie du Trocadéro, disposant à l'époque d'un fonds amazonien 'pauvre' alors que son directeur Paul Rivet est en quête matérielle de collections pour répondre à ses ambitions. En 1928, ce dernier est à 
Rio de Janeiro où il rencontre Jean Vellard, un scientifique français, à la fois diplômé en médecine et biologie, habitant et travaillant au Brésil. Paul Rivet apprécie "l'extraordinaire connaissance de la forêt tropicale " (Rivet apud Vellard 1939:7) et note l'intérêt du médecin biologiste pour partir sur le terrain. Jean Vellard devient correspondant pour le Muséum national d'Histoire naturelle, et propose en tant que tel un premier itinéraire dans l'Araguaya pour 'observer des populations Caiapo et Caraja'. Revenu de ce terrain en 1929, avec une collection de 280 objets et une liste de vocabulaire, il semble déçu de son résultat. Ce n'est pas le cas de Paul Rivet, qui le mandate par la suite sur trois autres campagnes de collecte à travers le continent sud-américain pour le compte du Musée de l'Homme. De ce fait, le nom de Jean Vellard est aujourd'hui lié à 1833 objets répartis en 4 collections.

Une autre collection du quai Branly, liée au nom de Vilma Chiara, est constituée de 126 objets incluant deux pièces kayapo et arrivés en même temps du Musée de l'Homme. Brésilienne, Vilma Chiara a étudié les sciences sociales à São Paulo, avant d'entrer comme stagiaire au Museu Paulista où elle est devenue conservatrice en 1960. Pour ce musée, elle a effectué de nombreuses campagnes de collecte avec son mari l'anthropologue Harold Schultz entre 1947 et 1965, et s'est chargée de l'inventaire et de la documentation des objets collectés. A partir de 1968, Vilma Chiara vit à Paris, étudie à l'Ecole des Hautes Etudes en Sciences Sociales (EHESS) et mène sa recherche au Musée de l'Homme avec une double casquette d'anthropologue et de muséologue (Batistella 2017). Avant sa thèse de doctorat, elle rédige un mémoire sur une collection du Musée de l'Homme constituée de poupées en céramique des Karaja, un peuple qu'elle connaît bien pour y avoir séjourné. Il est intéressant de noter que si cette collection est enregistrée en 1971 comme 'campagne de collecte de Vilma Chiara' avec une mention 'acquise sur le terrain en 1951', l'intéressée indique pourtant n'avoir rien collecté pour le Musée de l'Homme ${ }^{10}$. Dans ce cas, le nom resté attaché à la collection serait celui de la personne qui a documenté et non pas collecté les objets, mais toujours reconnue comme scientifique spécialiste. 


\section{L'essor des arts premiers au Musée du quai Branly, redéfinition des discours et réseaux}

En 2006, les collections ethnographiques du Musée de l'Homme sont transférées au Musée du quai Branly, nouvelle entité muséale dédiées aux arts et cultures extra-occidentales. En affirmant une politique de reconnaissance, les collections jusqu'alors vivent une transformation ambigüe de leur statut pour être intégrées à part égale dans l'histoire de l'art. Le fond hérité est donc redéfini selon le prisme de l'art et de l'esthétique. La loi relative aux musées de France est désormais en vigueur, les acquisitions de collections se font en majeure partie par le biais du marché de l'art (Doyen 2015 ; Delpuech 2015 ; Viatte 2008).

La galeriste Roberta Rivin est liée à la plus grande collection kayapo en termes numériques conservée au Musée du quai Branly. Après des études en anthropologie, cette américaine voyage dans le cadre de missions humanitaires dans les années 60 en Amérique latine puis s'installe au Brésil où elle achète une maison à Recife, Etat du Pernambouc. En 1972, elle déménage à Paris et fonde la galerie Urubamba consacrée aux 'objets indiens des Amériques, anciens et contemporains'. Pour se fournir, elle effectue plusieurs voyages sur le continent américain, assortis de séjours annuels chez elle au Brésil. Si elle a effectué des séjours chez les Karaja et les Ka'apor dans sa jeunesse, elle a mené par la suite ses achats en ville à des intermédiaires brésiliens, amérindiens ou non. Passionnée par les objets du quotidien (paniers, hamacs...) ou faits pour un usage interne, elle atteste constituer une collection sans logique artistique ou scientifique précise mais destinée à satisfaire sa clientèle parisienne ${ }^{11}$.

En 2001, Roberto Rivin a exposé des objets de sa galerie à la Mona Bismarck Foundation annonçant par la même occasion la vente de sa collection. Pour le futur Musée du Quai Branly, cet « ensemble remarquable, en état exceptionnel, de grande diversité, très spectaculaire [... ${ }^{12}{ }$ est une occasion sachant qu'une "plus grande place muséographique consacrée à l'Amazonie " sera accordée et que l'actuel fond amazonien de 
plumes est pauvre. La nouvelle institution achète directement une partie de la collection à Roberta Rivin en 2008 avec 203 objets issus de nombreuses régions brésiliennes suivie d'une seconde en 2010 de 121 objets. Tous ces objets venus d'Amazonie brésilienne sont désormais liés au nom de Roberta Rivin-Schuldenfrei, galeriste.

\section{Le Muséum de Toulouse, un fonds très ancien renouvelé par la Mission Brésil}

De toutes les collections inventoriées, la plus ancienne est conservée au Museum de Toulouse. Il s'agit d'un legs effectué en 1993 par la famille d'un missionnaire dominicain qui était parti s'installer avec les Kayapo au début du XX' $\mathrm{XX}^{\circ}$ siècle. Le Père Carrerot a accompagné la fondation puis le développement de Conceiçao do Araguaia, une mission consacrée à l'évangélisation des amérindiens, notamment Kayapo et Karaja (Chaves 2012 ; Pic 2014). Pendant plusieurs années, il a voyagé dans les états du Goias et du Para pour tenter de sédentariser différents groupes amérindiens dont les Tapirapé avant d'être sacré évêque, en 1911, à Toulouse. C'est à l'occasion de ce seul voyage de retour en France qu'il a du ramener les 23 objets donnés par la suite au Museum de Toulouse. Après expertise en collaboration avec des Mebêngôkre et durant les ateliers COLAM, il s'avère que ces objets - principalement des armes identifiées comme Cayapo ou Tapirapé au Museum - auraient été fabriqués par des Irã Ãmrãnh, un sous-groupe kayapo considéré comme disparu (Chaves 2012). C'est aujourd'hui au nom de ce missionnaire français devenu évêque en Amazonie brésilienne que la collection reste attachée.

A Toulouse, la collection ethnographique du muséum d'histoire naturelle compte 6.000 objets, dont $20 \%$ vient d'Amérique ('Amazonie est faiblement présente). En termes de politiques d'acquisition, Sylviane Bonvin-Pochstein, a fait le pari du retour à la collecte rompant ainsi avec le marché de l'art qu'elle qualifie de " non-raisonné et sans pertinence scientifique " (nécessaire dans le cas d'un Muséum). Son projet 'Mission Brésil'13 monté avec le photographe Serge Gui- 
raud $^{14}$, apprécié du secteur muséal ${ }^{15}$ pour sa connaissance du milieu amazonien et des cultures matérielles amérindiennes, et avec l'ethnologue Nathalie Petesch ${ }^{16}$ associée dans cette entreprise à d'autres spécialistes de la région, a été soutenu par les élus municipaux ${ }^{17}$. Au Brésil, des communautés amérindiennes déjà engagées dans des expériences liées à la transmission patrimoniale ont accepté de devenir partenaires du projet. Ainsi, de 2010 à 2016, six missions de collecte et d'échange ont été organisées par le Muséum: chez les Ina-Karajá de l'île de Bananal (Etat du Tocantins), chez les Yawalapiti du PIX (Etat du Mato Grosso), chez les Tapirapé de Urubu Branco (Etat du Mato Grosso), chez les Awaete-Asurini (Etat du Para), chez les Trumai (Mato Grosso) et chez les Kayapó (Etat du Pará). A chaque fois, une thématique de collecte est définie en partenariat avec les habitants d'un village puis la campagne se développe autour d'ateliers filmés consacrés à un savoir-faire donné et séances de formation à la vidéo pour les jeunes.

Pour cette dernière mission par exemple, le Museum a travaillé avec le village de Motukore par l'intermédiaire de Bepkamrek Kayapó, jeune leader déjà en charge de plusieurs projets culturels. Sylviane Bonvin-Pochstein, intéressée par la production contemporaine et l'introduction de nouveau matériaux comme le plastique dans l'artisanat, a collecté 125 objets documentés avec en collaboration avec l'anthropologue Nathalie Petesch et les habitants.

La collection issue de la 'Mission Brésil' comptabilise au total 500 objets contemporains, ainsi que des milliers de fichiers photos, et enregistrements sonores, soit une base de données importantes pour et sur ces populations. Si cette collection est le plus souvent nommée comme issue de la 'Mission Brésil', elle reste quand même attachée au nom d'un collecteur, en l'occurrence une collectrice, en la personne de Sylviane Bonvin-Pochstein. Toutefois, la plupart des objets réunis là sont également attachés à un nom d'auteur, celui de la personne qui l'a fabriqué. De cette manière, avec ces collections récentes, l'objet fait rentrer au musée les 
noms des personnes du village amérindien associé au Museum de Toulouse dans ce projet.

\section{La figure de l'expert au sein de la documentation}

Jusqu'aux années 80 au Musée de l'Homme, la majeure partie de ceux qui constituent des collections suivent les principes édictés par Marcel Mauss (1926). Quand l'objet arrive au musée, les notes constituées lors de la collecte sont converties au sein de la fiche à 10 points. Le nom de ceux qui rédigent ces fiches demeure souvent inconnu, ce qui peut être expliqué par un manque de considération déjà noté chez Marcel Mauss :

"L'idéal serait qu'une mission ne parte pas sans son géologue, son botaniste et ses ethnographes. On réduirait ainsi les frais généraux ; d'autre part, un anthropologue peut se révéler sociologue et tout le monde peut être excellent muséographe " (Mauss 1926:16).

Cette tache relève de la muséographie alors définie comme une sous-branche de l'ethnologie descriptive : «La muséographie d'une société consiste à établir les archives matérielles de cette société, les musées sont des archives " (Mauss 1926:16). Un discours à caractère ethnographique est attribué à l'objet-témoin. Transcrit par un système de liste réduisant les possibilités d'interprétation (Dias 1988), il participe à l'établissement d'un regard figé sur la société représentée et à " la réduction des multiples récits " que suscite toujours chacun de ces objets à « une narration unique » et exclusive (Oliveira \& Santos 2019:16).

L'objet, perçu comme une archive, est considéré au passé alors qu'il reflète une société en constante redéfinition. Avec une initiative comme celle du Museum de Toulouse, de nouveaux champs apparaissent comme le nom de 'l'auteur' dès lors qu'il est connu. La documentation d'un objet peut donc nommer les 'faiseurs de collections', le producteur de l'objet et/ou le rédacteur de la documentation en question. Ces informations induisent une dimension personnelle, institutionnalisée par le musée. 


\section{Conclusion}

Renseigner l'histoire des collections permet de révéler les récits portés sur les objets de musée et ainsi les possibilités de dialogues autour de ces objets tout en soulignant leurs enjeux. La contextualisation et l'approche comparative aide à comprendre comment les politiques muséales sont mises en œuvre pour répondre aux prérogatives des institutions comme aux injonctions externes. Les pratiques patrimoniales sont tenues par un lien étroit entre les dimensions institutionnelles et personnelles. L'enrichissement des collections, aussi bien matériel de par l'acquisition ou immatériel via la documentation, doit être pensé comme un acte d'alliance et d'échange mais également comme une responsabilité devant être réfléchie avec l'ensemble des partenaires impliqués, et sur le long terme.

Les personnes-clés à l'origine de l'entrée de la collection au musée, autant que les réseaux dans lesquels ils évoluent, influencent la constitution du patrimoine en termes d'inclusion et de conservation de savoirs. Nommés dans la documentation des collections, ils font office d'experts. Ces processus de légitimation mis en lumière initient une réflexion sur la place et l'intégration des savoirs qu'ils représentent au sein de l'institution. S'il n'est pas aujourd'hui textuellement question de savoirs associés aux collections, ni de reconnaissance de voix autochtones, il sera intéressant de suivre les évolutions possibles pour les politiques d'acquisition et de conservation faisant face aux normes internationales, comme le protocole de Nagoya (Aubertin \& Nivart 2017). Cette perspective, en revanche, est prise en compte dans le cadre de projets de documentation des collections effectués en collaboration avec des populations indigènes héritières du patrimoine étudié. Ce fut le cas dans le cadre du projet de recherche COLAM pour lequel la recherche présentée au travers de cet article a permis de proposer un matériel de travail pertinent à documenter selon les points de vue des partenaires du projet. Tenu en 2018, l'échange mis en place entre experts amérindiens, muséologues et scientifiques autour d'objets à la documentation lacunaire a permis de l'enrichir et 
la réactualiser. Si la documentation accompagne l'objet dans sa vie au musée, elle est comme lui 'immortalisée' (dans la mesure du possible, là est le paradoxe du musée). Si elle ne peut être détruite, elle peut ainsi être revue sous un jour nouveau. Les discours et visions qui s'y superposent au fil de la vie des objets au musée peuvent ainsi apparaître comme la base de dialogues futurs à instaurer au sein de l'espace muséal. Il s'agit, autant que faire se peut, de retrouver et de redonner à voir et à entendre, les multiples récits attachés à chacun des objets conservés au Musée.

\section{Notas:}

1 O projeto 'COLAM - Coleções dos Outros e memórias de encontros: objetos, plantas e histórias da Amazônia' se relaciona a três linhas principais de reflexão em torno de coleções museológicas a partir de exemplos amazônicos: 1) a renovação dos estudos sobre coleções e conhecimento associado ; 2) o desenvolvimento de novas formas e éticas de pesquisa que integram todos os atores das pesquisas ; 3 ) o impacto das políticas ambientais e culturais e da legislação internacional sobre os patrimônios. Projeto OPUS/Sorbonne Université, Parceiros IRD-PALOC Paris / CCH-MPEG Belém/NEPE-UFPE Recife. (www.institut-opus.fr/aap-2017-2018-retour-sur-le-projet-colam/).

2 Sous la tutelle de Pascale de Robert (IRD) et de Lucia van Velthem (MPEG), le travail de recherche a été mené dans le cadre du Master de Muséologie du MNHN Paris avec une bourse du laboratoire PALOC (IRD).

${ }^{3}$ En témoigne la polémique qui a suivi la sortie en novembre 2018 du rapport Sarr/ Savoy 'Restituer le patrimoine africain : vers une nouvelle éthique relationnelle'.

4 Parmi les ouvrages collectifs les plus récents sur ces questions au Brésil, on peut consulter notamment (Françozo et al 2017; Athias \& Gomez 2018; Oliveira \& Santos 2019).

5 Ces premières aires d'étude ont été choisies car le projet COLAM1 est lié au projet de recherche IRD/CNPQ 'Systèmes agricoles localisés dans une agriculture globalisée : transformation, émergence et connectivité - PACTA III' qui œuvre avec des populations traditionnelles brésiliennes sur la question de la diversité bio-culturelle. Les collections (botaniques et ethnographiques) y apparaissent comme motif de rencontre et d'échange autour des politiques de revalorisation patrimoniale.

6 Un inventaire général des collections américanistes en France doublé d'un travail de renseignement de l'histoire des collections a été réalisé par des chercheurs et étudiants de l'institut d'Art et d'Archéologie (Sorbonne Université) de 1976 à 1999. 
7 Les ethnonymes et toponymes associés aux collections ethnographiques sont de précision et d'orthographe très variables. Pour ce qui concerne cette recherche, nous avons consulté les collections associées aux termes kayapo, caiapo, gorotire, mebengokre, kuben kran ken, xikrin, txikrin, rio araguaia, etc

8 L'Appellation 'Musée de France' peut être accordée aux musées appartenant à l'État, à une personne morale de droit public ou de droit privé à but non lucratif. À ce jour, 1218 musées ont reçu cette appellation.

9 Mention de la mission dans les lettres des 06/10/1928, 13/11/1929, $15 / 01 / 1930,11 / 12 / 1930$ et 21/04/1933. Correspondance avec P. Rivet. Vega-Vent (2 AP 1 C21a). Archives conservées à la bibliothèque du MNHN

10 Déclaration faite par l'intéressée lors d'échange par mail avec Anouk Delaître en 2017.

11 Entretien avec un ami et client de la galeriste, Carnet de terrain de Anouk Delaître (2017).

12 Archives du Musée du quai Branly D000579/32900, Avis sur objet en plumes (11/06/2002). D000579/32901. Proposition d'objets (27/08/2002). D000579/32912. Notes concernant la présentation des objets au comité consultatif (10/01 - 13/01/2003) (04/04/2003 17AA/73).

13 https://www.museum.toulouse.fr/mission-bresil.

14 Il est à la tête de l'association Jabiru prod axée sur la valorisation de l'Amazonie traditionnelle par le biais du projet Alapi offrant aux populations locales des formations aux techniques de la vidéo et du cinéma, un mode d'expression courant chez les Amérindiens.

15 Il a participé, entre autres, à l'expertise de collections au muséum de Lille, celui de La Rochelle, et à charité de Marseille. A Toulouse, il est chargé par le Muséum d'acquisition, de prêt, d'expertise et de don iconographique.

16 Membre de l'équipe de recherche en ethnologie amérindienne du CNRS (UPR 324).

17 Le Muséum de Toulouse fonctionne en régie directe avec la Métropole de Toulouse.

\section{Reférences:}

APPADURAI, Arjun (ed.). 1986. The social life of things. Commodities in cultural perspective. Cambridge: Cambridge University Press.

ATHIAS, R. \& GOMES, A. (ed.). 2018. Coleções Etnográficas, Museus Indígenas e Processos Museológicos. Recife: Editora da UFPE.

AUBERTIN, C. \& NIVART, A. 2017. "Musée et collections sous le protocole de Nagoya". In MAIRESSE, F. (ed.): Définir le musée du XXIe siècle. Matériaux pour une discussion, pp.133-137. Paris: International Commitee for Museology ICOM 
BATISTELLA, Aline M. 2017. Experiências etnográficas de Harald Schultz e Vilma Chiara entre os povos indigenas. Dissertação de Mestrado. Cuiabá: Universidade Federal do Mato Grosso.

BONVIN-POCHSTEIN, Sylviane. 2018. Projet Brésil Central : constitution des collections aujourd'hui. Un exemple de collaboration. Trabalho apresentado no "Colloque International Constitution, documentation et valorisation des collections muséales en collaboration. Pratiques d'hier, aujourd'hui et demain", MHNT/IRD. Toulouse - France.

BELTRAME, Tiziana N. 2012. "Un travail de Pénélope au musée. Décomposer et recomposer une base de données". Revue d'Anthropologie des Connaissances, $6(1): 217-237$.

BENSA, Alban. 2006. La fin de l'exotisme, essais d'anthropologie critique. Toulouse: Éd. Anacharsis.

BLANCKAERT, Claude. 2015. Le Musée de l'Homme Histoire d'un musée-laboratoire. Paris: Muséum national d'Histoire naturelle/Artlys.

BOLTANSKI, L. \& ESQUERRE, A. 2017. “'La collection'. Une forme neuve du capitalisme. La mise en valeur du passé et ses effets”. Les Temps modernes, 679: $5-72$.

BONNOT, Thierry. 2014. L'attachement aux choses. Paris : CNRS éditions.

BUCHILLET, Dominique. 1993. "Droits constitutionnels et démarcation des terres au Brésil”. Journal de la Société des Américanistes, 79:225-231.

CHAVES, Carlos E. 2012. Nas trilhas Irã Ãmrãnh sobre história e cultura material Mebêngôkre. Dissertação de Mestrado. Belém : Universidade Federal do Pará.

CLIFFORD, James. 1997. Routes, Travel and translation in the late twentieth Century. Cambridge: Harvard Press.

CODE DU PATRIMOINE. 2002. Livre IV : musées, Titre IV : régime des musées de France. Loi n 2002-5 du 4 janvier 2002 relative aux musées de France.

DELAITRE, Anouk. 2017. Derrière les collections ethnographiques. Inventaires et mises en récits de collections ethnographiques. Mémoire de Recherche. Paris: MNHN.

DE L'ESTOILE, Benoit. 2007 [2010]. Le Goût des Autres: De l'exposition coloniale aux Arts premiers. Paris: Flammarion.

DELPUECH, André. 2015. "Actualité et débats autour du marché de l'art amérindien » in Lettre du Comité français de l'ICOM, n³8, « Déontologie des collections publiques : intérêt général et acteurs privés ». Deuxièmes journées d'études, 25-26 novembre 2013, Paris. Comité national français de l'ICOM : pp.15-26.

DIAS, Nélia. 1988. "Vers l'archivage des objets: la naissance du Musée d'ethnographie du Trocadéro". Bulletin d'Information de l'Association des Bibliothécaires Français, 138:28-31.

DOYEN, Audrey. 2015. "L'objet ethnographique entre marché et patrimoine : cartographie de quelques enjeux". ICOFOM Study Series, 43:95109.

FRANÇOZO, Mariana et al (eds.). 2017. "Dossiê: Patrimônio indígena e coleções etnográficas”. Bol. Mus. Para. Emílio Goeldi. Ciênc. Hum, 12(3):709-711. 
FERNANDO BANIWA, André. 2018. Sobre visita nos museus de Paris, France. Rapport de Mission de Recherche, Projet COLAM, IRD-Paloc. Paris.

GROGNET, Fabrice. 2013. "La réinvention du musée de l'Homme au regard des métamorphoses passées du Trocadéro". In MAZE, C., POULARD, F. \& VENTURA, C. (eds.): Les musées d'ethnologie. Culture, politique et changement institutionnel, pp. 37-70. Paris: CTHS.

GRUPIONI, Luis D. 1998. Coleções e Expedições Vigiadas: os etnólogos no Conselho de Fiscalização das Expedições Artísticas e Científicas no Brasil. São Paulo: Hucitec/ ANPOCS.

MAUSS Marcel. 1926 [1967]. Manuel d'ethnographie. Paris: Éditions Sociales.

MONGNE, Pascal. 2013. "Les collections amérindiennes de France : l'histoire d'une image ou le balancier du goût”. In Havard, G. \& Augeron, M. (eds.): Un continent en partage. Cinq siècles de rencontres entre Amérindiens et Français, pp. 455-464. Paris: Les Indes savantes.

1999. Inventaire des collections amérindiennes au sein des musées français. Note de recherche non publiée.

OLIVEIRA, J. P. \& MELO, R. C. (eds.). 2019. De acervos coloniais aos museus indígenas: formas de protagonismo e de construção da ilusão museal. PB: Editora da UFPB.

ORGANISATION Internationale des Musées. 2006. Code de déontologie de l'ICOM pour les musées. (http://archives.icom.museum/ethics_fr.html>; acesso em 12/12/2019).

. 1946. Statuts de l'ICOM. ( http://icom.museum/fileadmin/user_upload/ pdf/Statuts/2016_Statutes_FR.pdf; acesso em 12/12/2019).

PIC, Claire. 2014. Les dominicains de Toulouse au Brésil (1881-1952): de la mission à l'apostolat intellectuel. Thèse de Doctorat. Toulouse: Université de Toulouse le Mirail - Toulouse II.

PIERREBOURG, F. \& SEVILlA, C. 2010. "Recuerdos de América a Francia: el acervo etnografico del musée du Quai Branly”. In RUZ, M. H. \& TEMPLE SELLEN, A. (ed.): Las vitrinas de la memoria, los entresijos del olvido. Coleccionismo e invención de memoria cultural, pp. 15-55. Mérida: UNAM.

POMIAN, Krzysztof. 1996, "Histoire culturelle, histoire des sémiophores". In RIOUX, J.-P. \& SIRINELLI, J.-F. (eds.): Pour une histoire culturelle, pp. 73-100. Paris: Seuil.

RIVET, Paul. 1939. "Préface". In VELLARD, J. (ed.): Une civilisation du miel. les Indiens Guayakis du Paraguay, pp. 5-6. Paris: Gallimard.

SOULIER, Virginie. 2013. Donner la parole aux autochtones: Quel est le potentiel de reconnaissance de l'exposition à plusieurs points de vue dans les musées?. Thèse de Doctorat. Avignon: Universités du Québec à Montréal et d'Avignon/ Pays de Vaucluse.

UNEP/CDB/COP/10. 2010. Nagoya Protocol on Access to Genetic Resources and the Fair and Equitable Sharing of Benefits Arising from their utilization. Decision as adoped (advanced unidited version). 
VIATTE, Germain. 2006. Tu fais peur tu émerveilles. Musée du Quai Branly, acquisitions 1998/2005. Paris: Réunion des Musées Nationaux.

Résumé: En amont d'une expertise de collections par des représentants Mebêngôkre-Kayapó, un inventaire a été mené aux Musée du Quai Branly et Muséum de Toulouse pour identifier des pièces à la documentation lacunaires. L'analyse de la documentation des collections doublée par celle de l'histoire des collections est une opportunité d'étude comparative et chronologique sur les politiques d'enrichissement concernant les deux musées. Des personnage-clés, ancré dans des réseaux déterminés et déterminants, sont identifié dans la constitution des collections. Si l'on découvre une multiplicité de regards et de savoirs chaque fois légitimés par l'entrée des collections qu'ils accompagnent au musée, dans quelle mesure les réseaux développés lors d'acquisitions de collections l'ont-ils influencé ? A quelle époque? dans quels objectifs? Ce cas amazonien illustre les processus de légitimation opérés par l'institution muséale française en contextualisant les conditions d'acquisition d'un point de vue institutionnel, politique et juridique.

Mots-clés: Muséologie, Collections ethnographique, Amérindiens, Documentation participative, Étude de provenance, Politique d'acquisition. 